\title{
Rouard's method as a modelling tool for the sensing characteristics of complex fibre Fabry-Perot interferometers formed between chirped fibre Bragg gratings.
}

\author{
Edmon Chehura, Stephen W. James, and Ralph P. Tatam* \\ Optical Sensors Group, Centre for Photonics and Optical Engineering, School Engineering, \\ Cranfield University, Cranfield, Bedford, MK43 0AL, UK.
}

\begin{abstract}
Phase and spectral response characteristics of fibre Fabry-Perot interferometers (FFPI), formed between linearly chirped fibre Bragg gratings (FBG), were theoretically modelled using Rouard's method. The method models accurately the phase shift through a complex grating structure which the transfer matrix model is unable to achieve. The FBG FFPI cavities were modelled with variable free spectral range that was tailored further to provide strain insensitivity and strain enhancement by making the chirp parameter and cavity length satisfy the desired conditions at a given wavelength of illumination.
\end{abstract}

Keywords: Fibre Bragg grating, chirp, Rouard's method, Fibre Fabry-Perot, sensors, strain

\section{INTRODUCTION}

The advent of fibre Bragg grating (FBG) technology has triggered a wide range of applications in sensors, fibre lasers, wavelength division multiplexing and demultiplexing for optical communication, pulse compression, and spectral filters among other applications ${ }^{1}$. Sensor development often benefits from designs based on theoretical modelling to achieve desired sensor characteristics. Most techniques are based on solving coupled mode differential equations for uniform FBGs and the transfer matrix model (TMM) for non-uniform FBGs ${ }^{2}$.

This paper describes the adaptation and implementation of the well established Rouard's method, a recursive technique largely used for thin film coating design ${ }^{3,4}$, for modelling complex chirped FBG-based fibre Fabry-Perot interferometers (FFPI) for sensing applications. The model is used to track accurately the phase shift of light propagating through the complex grating structure, critical to sensor design, which the TMM is unable to do. The TMM fails in this application because of the inherent coupled mode theory assumption that requires a grating section to be subdivided into equal sized elements of constant optical parameters which causes a linearly chirped grating to behave as a step chirped grating.

Rouard's method is in this paper applied to FBG FFPI structures that are tailored by linear chirp to produce variable free spectral range (FSR) that provides a large dynamic measurement range with variable resolution and sensitivity. Two such cavities are shown here to further provide strain insensitivity or sensing enhancement for particular chirp parameters and cavity lengths ${ }^{6}$. Other potential applications include the use of a cavity with a small FSR to provide mode-locking of short pulse fibre lasers or the utilisation of a cavity with a large FSR as optical filters for noise rejection.

\section{FBG THEORY}

An FBG is a periodic modulation of the refractive index within the core of an optical fibre and acts to couple a forward propagating fibre core mode to a backward propagating core mode at a particular wavelength known as the Bragg wavelength.

Chirped FBGs are formed by varying the grating period $\Lambda(z)$ along the length $L$ of the grating such that it varies from $\Lambda(0)$ at the beginning, to $\Lambda(L)$ at the end of the grating. The reflected Bragg wavelength $\lambda_{B}(z)$ is thus a function of length along the grating and the resulting spectrum becomes broadened in wavelength. The period of a linearly chirped FBG is described by equation 1 .

\footnotetext{
${ }^{*}$ r.p.tatam@cranfield.ac.uk, phone 441234 754630; fax 441234 752452; http://www.cranfield.ac.uk/sme/cpoe
} 


$$
\Lambda(z)=\Lambda(0)+\frac{(\Lambda(L)-\Lambda(0))}{L} \cdot z
$$

The refractive index modulation within the grating is generally sinusoidal. This paper considers the average refractive index within half of a period, which is equivalent to a square index modulation within the period of the grating. Each period is divided into two equal lengths, one with high and the other lower average refractive index. The amplitude of the $a c$ refractive index modulation used in obtaining the results reported in this work is $6 \times 10^{-4}$ and the entire FBG was apodised to remove side lobes and higher frequency ripples using a Gaussian function. These parameters resulted in reflectivity of $\sim 7 \%$ over a chirp bandwidth of $100 \mathrm{~nm}$ for a single FBG. The reflectivity chosen here is typical of experimentally achievable values for wide bandwidth chirped gratings.

\section{PRINCIPLES OF ROUARD'S METHOD}

Rouard's analysis was applied to 5 FBG FFPI cavities of differing configurations illustrated in Figure 1 and sample results are reported. Figure 1(a) shows a cavity constructed using uniform period FBGs. The chirped cavities are formed between 2 linearly chirped FBGs of either opposite chirp such that the chirp of both decreased in the direction of the cavity (figure 1(b)), or outward (figure 1(d)), or same sign chirp (figures 1(c) and 1(e)). The centre to centre cavity length between FBG1 and FBG2, each $4 \mathrm{~mm}$ long, was set to $124 \mathrm{~mm}$ while the chirp rate of the period was fixed at $25 \mathrm{~nm} / \mathrm{mm}$. These parameters are such that the optical path length of light at a wavelength of $1550 \mathrm{~nm}$ propagating through the chirped FBG FFPI structure of Figure 1(b), in the absence of perturbations, is balanced to its path length in the presence of axial strain perturbations. An analytical expression has previously been reported to describe this condition ${ }^{6}$. The parameters of this design condition were used on all 5 cavities and it was shown that the FFPI cavity of Figure 1(b) was insensitive to strain while the rest remained sensitive.

Figure 2 illustrates the underlying principles of Rouard's method that was applied in this work. The diagram has been simplified for clarity to show only three consecutive periods $\Lambda(i-1), \Lambda(i)$, and $\Lambda(i+1)$ of the FBG, where $i$ varies from 1 to a total of $N$ periods. Each period has been subdivided into two equal layers, shown by regions $n$ and $p$, with low and high refractive index respectively.

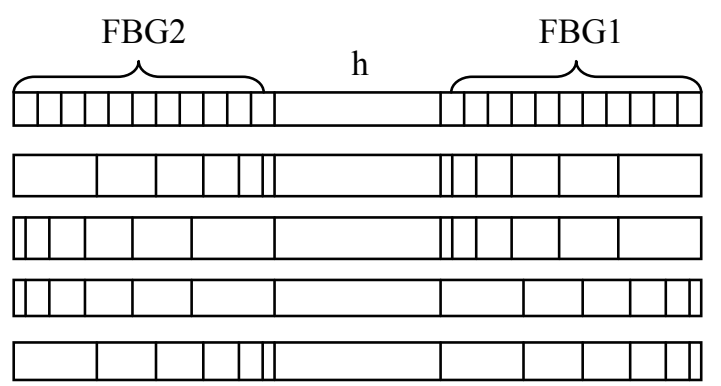

Figure 1: FBG FFPI configurations that were investigated (a) uniform FBGs, (b)-(e) chirped FBGs. FBG1 and FBG2: FBGs; h: physical separation between FBGs.
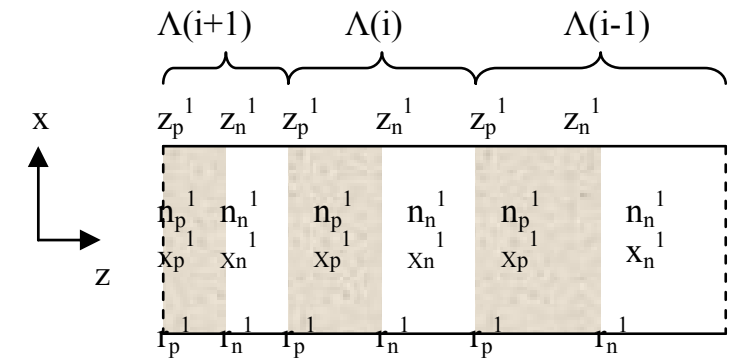

Figure 2: A section of a chirped FBG showing three consecutive periods that are subdivided into half periods to define thin layers, required to apply Rouard's method.

The following parameters are defined for the $i^{\text {th }}$ period of FBG1. The period $\Lambda(i)$ is given by the sum of the two layers, $x_{p}{ }^{l}(i)$ and $x_{n}{ }^{l}(i)$. The 1 in the superscript denotes FBG1. The refractive indices in layers $p$ and $n$ are $n_{p}{ }^{l}(i)$ and $n_{n}{ }^{l}(i)$ respectively. The reflection coefficient at the interface between layers $n$ and $p$ within this period is $r_{n}{ }^{1}(i)$. The interface between layer $p$ of this period and layer $n$ of the next period $\Lambda(i+1)$ has a reflection coefficient of $r_{p}{ }^{l}(i)$. Similar notation is followed in describing the parameters of FBG2 at the input side of the FFPI structure.

Equation 2 evaluates the Fresnel reflection coefficient at the interface between layers $n$ and $p$ within the $i^{\text {th }}$ period of FBG1 while the reflection coefficient at the interface between the $i^{\text {th }}$ and $(i+1)^{\text {th }}$ periods is given by $r_{p}{ }^{l}(i)$ in equation 3. The phase shift occurring on traversing layers $p$ and $n$ of the $i^{\text {th }}$ period of FBG1 is calculated from equations 4 and 5 respectively, by assuming normal incidence in the grating period.

$$
r_{n}{ }^{1}(i)=\frac{n_{p}{ }^{1}(i)-n_{n}{ }^{1}(i)}{n_{p}{ }^{1}(i)+n_{n}{ }^{1}(i)}
$$

$$
r_{p}{ }^{1}(i)=\frac{\left.n_{n}{ }^{1}(i+1)\right)-n_{p}{ }^{1}(i)}{n_{n}{ }^{1}(i+1)+n_{p}{ }^{1}(i)}
$$




$$
\phi_{p}{ }^{1}(i)=\frac{2 \pi \cdot n_{p}{ }^{1}(i) \cdot x_{p}{ }^{1}(i)}{\lambda}
$$

$$
\phi_{n}{ }^{1}(i)=\frac{2 \pi \cdot n_{n}{ }^{1}(i) \cdot x_{n}{ }^{1}(i)}{\lambda}
$$

The effective complex reflectance $\rho_{p}{ }^{l}(i)$ for all structures from the output end of the FFPI up to and including the interface between the $i^{t h}$ and $(i+1)^{t h}$ periods of FBG1 is determined from the Airy function, equation (6). Similarly, equation 7 is an Airy function that provides the effective complex reflectance at the interface between regions $n$ and $p$ within the $i^{\text {th }}$ period of FBG1, for the combination of all structures from the output end up to and including this interface.

$$
\rho_{p}{ }^{1}(i)=\frac{r_{p}{ }^{1}(i)+\rho_{n}{ }^{1}(i) \cdot \exp \left[-2 j \phi_{p}{ }^{1}(i)\right]}{1+r_{p}{ }^{1}(i) \cdot \rho_{n}{ }^{1}(i) \cdot \exp \left[-2 j \phi_{p}{ }^{1}(i)\right]}
$$

$$
\rho_{n}{ }^{1}(i)=\frac{r_{n}{ }^{1}(i)+\rho_{p}{ }^{1}(i-1) \cdot \exp \left[-2 j \phi_{n}{ }^{1}(i)\right]}{1+r_{n}{ }^{1}(i) \cdot \rho_{p}{ }^{1}(i-1) \cdot \exp \left[-2 j \phi_{n}{ }^{1}(i)\right]}
$$

Equations 3-8 are recursive and they form the basis of Rouard's method. The method is applied to the first layer (substrate) to determine the reflection coefficient at its interface and is extended to the next layer to determine the effective complex reflectance at that interface. The approach is continued until the reflectance at the input end interface of the cavity is determined. The effective complex reflectance $\rho_{n}{ }^{2}(N)$ at the input interface, from the whole FFPI structure is therefore given by the Airy function of equation 8 . The Fresnel reflection coefficient at the input end of the FFPI is denoted by $r_{n}{ }^{2}(N)$ while superscript 2 represents FBG2 and $N$ is the total number of periods in this grating. The required reflectivity and phase response of the FBG FFPI structure are evaluated from this equation.

$$
\rho_{n}{ }^{2}(N)=\frac{r_{n}{ }^{2}(N)+\rho_{p}{ }^{2}(N) \cdot \exp \left[-2 j \phi_{n}{ }^{2}(N)\right]}{1+r_{n}{ }^{2}(N) \cdot \rho_{p}{ }^{2}(N) \cdot \exp \left[-2 j \phi_{n}{ }^{2}(N)\right]}
$$

\section{DISCUSSION}

Figure 3 shows the FSR variations over $100 \mathrm{~nm}$ for the chirped FBG FFPI cavities (b)-(e) of Figure 1 with the same labels used to denote the curves. All the cavities were modelled at the condition which makes the cavity of Figure 1(b) insensitive to axial strain as discussed. The cavity that was designed to be insensitive to axial strain exhibited large FSR values than other cavities. The smallest FSR values were obtained with the cavity of Figure 1(d) and the density of fringes was therefore largest compared to the other cavities and the cavity is therefore most sensitive to axial strain. All cavities, as expected, had their largest FSR values at the design wavelength of $1550 \mathrm{~nm}$. These results serve to show the versatility of the chirped FBG FFPI cavity sensors in their ability to vary in wavelength resolution, sensitivity, and measurement dynamic range. The large FSR at $1550 \mathrm{~nm}$ can be utilised for noise rejection as an optical filter.

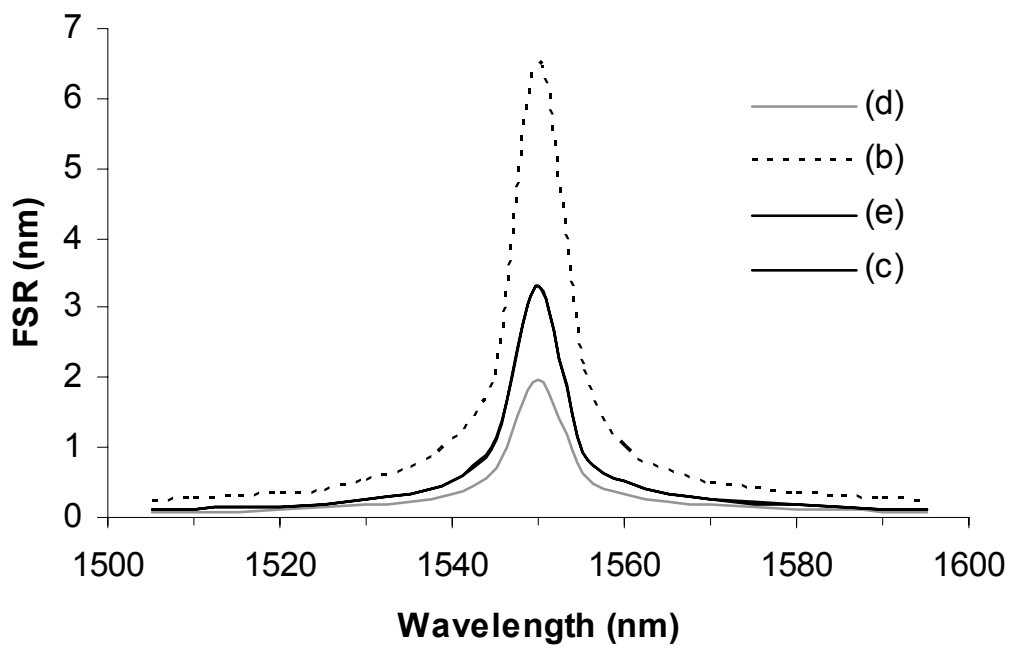

Figure 3: Free spectral range (FSR) variation with wavelength for FBG FFPI configurations of Figure 1(b)-(e). Curves (c) and (e) are indistinguishable. 
Figure 4 shows the reflectivity spectrum of the cavity of Figure 1(b) designed such that it is insensitive to strain at the wavelength of $1550 \mathrm{~nm}$ as discussed. The effect of axial strain was included into the model and no change was found in the spectral response centred at $1550 \mathrm{~nm}$ indicating that this cavity configuration is insensitive to axial strain at this wavelength. This behaviour was not observed in other cavities where fringes tended to sweep across the entire spectrum when strain was applied.

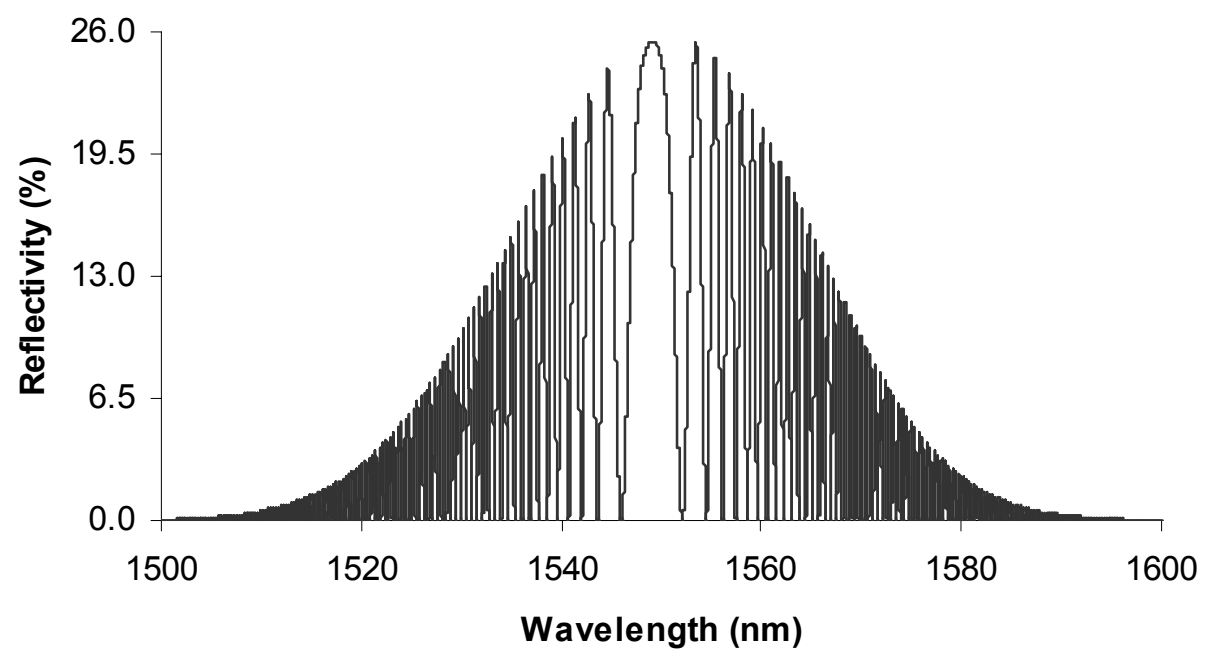

Figure 4: Reflectivity spectrum of the FFPI cavity of Figure 1(b).

The total phase response in reflection for this cavity was observed to be parabolic with a minimum at the wavelength of $1550 \mathrm{~nm}$, corresponding to the wavelength with the largest FSR.

\section{CONCLUSION}

Rouard's method has been used to model accurately the sensing characteristics of FFPI cavities formed by 2 linearly chirped FBGs in a single mode optical fibre. It was observed that chirped FBG FFPI cavities exhibit variable FSRs that can be utilised to provide variable resolution, sensitivity and dynamic range in sensor measurement applications. One such cavity demonstrated insensitivity to strain when its chirp parameters and cavity length were chosen so as to equalise the optical path length in the presence and absence of axial strain loading.

\section{ACKNOWLEDGEMENTS}

The work was supported by the Engineering and Physical Sciences Research Council, UK under grant No. GR/T09149/01.

\section{REFERENCES}

1. Y. J. Rao, "Recent progress in applications of in-fibre Bragg grating sensors", Opt. Laser. Eng., 31, pp. 297-324, 1999.

2. T. Erdogan, "Fiber grating spectra", J. Lightwave Technol., LT-15, pp. 1277-1294.

3. L. A. Weller-Brophy, and D. G. Hall, "Analysis of waveguide gratings: application of Rouard's method", J. Opt. Soc. Am. A, 2, pp. 863-871, 1985.

4. Z. H. Wang, G-D. Peng, and P. L. Chu, "Improved Rouard's method for fiber and waveguide gratings", Opt. Comm., 177, pp. 245-250, 2000.

5. K. P. Koo, M. LeBlanc, T. E. Tsai, and S. T. Vohra, "Fiber-chirped grating Fabry-Perot sensor with multiple-addressable freespectral ranges", IEEE Photonic. Tech. L., 10, pp. 1006-1008, 1998.

6. C. S. Cheung, S. W. James, C-C. Ye, and R. P. Tatam, "The strain sensitivity of fibre Fabry-Perot cavities formed between chirped fibre Bragg gratings", 16th Int. Conf. on Optical Fiber Sensors (Nara, Japan), (Japan: IEICE), pp. 802-805, 2003. 